\title{
Evolución histórica del uso y abuso de MDMA
}

\author{
Pilar A. Sálz Martínez*, Paz García-Portilla González*, \\ Begoña Paredes Ojanguren**, Julio Bobes García*** \\ *Profesor Titular de Psicología Médica **Doctorando Area Psiquiatría ** Catedrático de Psiquiatría \\ Enviar correspondencia: Pilar Alejandra Sáiz Martínez. Area de Psiquiatría. Facultad de Medicina. \\ Julián Clavería $6-3^{\circ} .33006$ Oviedo (Asturias).Tfno/FAX: +34 98510 3552. E-mail: frank@correo.uniovi.es
}

\section{Resumen}

La MDMA (éxtasis), es sin duda, la droga que mayor popularidad ha alcanzado durante la última década. A pesar de haber sido sintetizada en 1912, no es hasta finales de los años 70 cuando comienza a ser utilizada por diversos grupos poblacionales en Estados Unidos (estudiantes, yuppies, gays y new agers). En el año 1985 es incluida en la Lista I de la Comprehensive Substances Act americana y en el año 1986, la OMS la incluye en la Lista I del Convenio sobre Psicotrópicos. Su posterior gran expansión a escala mundial se ha asociado a determinadas subculturas musicales y de ocio. En España, su difusión ha pasado por cinco fases diferentes: fase previa (1978-1986), fase inicial (1987-1989), fase de popularización (1990-1991), fase de vulgarización y consumo masivo (1992-1996) y fase de rutina y agotamiento del ciclo o fase de estabilización (1997-). Durante los últimos años existe cada vez mayor interés por parte de organismos internacionales y nacionales en la puesta en marcha de acciones conjuntas dirigidas a conocer la verdadera magnitud epidemiológica del fenómeno, sus consecuencias sobre la salud y en la puesta en marcha de acciones dirigidas a aminorar los potenciales daños asociados a su consumo.

Palabras claves: MDMA (éxtasis), aspectos históricos, aspectos legales, uso recreativo.

\section{Summary}

MDMA (ecstasy) has undoubtedly been the most popular recreational drug during the last decade. In spite of its having been synthesised in 1912 it was not employed as a drug by different groups in the United States until the end of the seventies (students, yuppies, gays, and new agers). In 1985 it was included in List I of the Amercican Comprehensive Substances Act, and in 1986, the WHO included it in List I of the Psychotropic Convention. Its greater spread on a worldwide bases has been associated with specific musical and recreational subcultures. In Spain, its spread has been over 5 different phases: previous phase (1978-1986), initial phase (1987-1989), popularity phase (1990-1991), phase of common use and masive consumption (1992-1996), and routine and end of cycle or stabilisation phase (1997-). During the last few years a considerable interest has been shown by international and national organisations to begin joint actions in order to know the real epidemiological magnitud of the phenomenon, its consequences on health, and the development of preventive actions to ameliorate the potential risks associated with its consumption.

Key Words: MDMA (ecstasy), historical aspects, legal aspects, recreational use.

\section{MDMA: BREVE RESEÑA HISTORICA}

B ajo el epígrafe "drogas de síntesis" o "drogas de diseño", término éste último acuñado por Gary Henderson, far- macólogo de la Universidad de California, durante los años sesenta, se agrupan una serie de sustancias de origen sintético que en esa época comenzaban a ser objeto de tráfico ilegal. En general son sustancias psicoactivas creadas a partir de la modificación 
de la estructura química de determinados productos naturales o de determinadas sustancias utilizadas como medicamentos, que en su mayor parte adoptan la forma de "pastillas", y que pueden ser fácilmente sintetizadas por métodos químicos sencillos.

Quizás la más conocida de todas ellas es la MDMA (3-4 metilenodioximentanfetamina), sustancia perteneciente al grupo de las feniletilaminas (de estructura parecida a la anfetamina, pero dotada de un anillo bencénico y grupo amino), más conocida vulgarmente como "Extasis" (aunque también puede adoptar otros nombres como "Adam", nombre con que la sustancia es especialmente conocida en América -en slang americano si MDMA se pronuncia velozmente, suena de este modo-, "E", simplemente su inicial en Inglaterra, "XTC", del mítico conjunto musical del comienzo de los 80 que producía música en un estado de conciencia modificado, " $X$ $X^{\prime \prime}$, que en inglés se pronuncia como exces, "Presencia", "Esencia", "Claridad", "Zen", "Doctor", "M\&M", "M", “Euforia", "Venus", "Banana Split,", "Fido Dido," "Simpson", “Disco Biscuit,", “Disco Burger," “Love Dove,, “New Yorker", "Power Thruster", “Cracker",...). Un gran número de nombres diversos que corresponden a efectos ligeramente diferentes, de más anfetamínicos a más psiquedélicos, en función de las distintas composiciones de las pastillas.

Aunque la popularidad y el reconocimiento a prácticamente todos los niveles, de la MDMA cuenta con algo más de una década de evolución, su verdadera historia es mucho más antigua, ya que fue sintetizada por vez primera en diciembre de 1912, en los Laboratorios de E. Merck en Darmstadt (Alemania), $y$ aunque existen numerosos informes que señalan que fue patentada dos años más tarde, en 1914 (patente $n^{\circ} 274.350$ ), en realidad, la sustancia patentada fue la MDA (3-4metilendioxianfetamina), sustancia de estructura prácticamente similar al éxtasis y que había sido sintetizada en 1910 por dos químicos, Mannish y Jacobsen, de la misma compañía farmaceútica. El interés de patentarla residía en la intención de introducirla en el mercado como fármaco anorexígeno, aunque nunca llegó a ser comercializada (Shulgin y Shulgin, 1992).

Posteriormente cayó en el olvido, hasta que en 1953 vuelven a tenerse noticias de ella, momento en que el ejercito norteaméricano la utilizó, junto con otras sustancias, bajo la codificación de agente experimental $n^{\circ} 1475$ (Holsten y Schieser, 1986), en estudios toxicológicos en animales, constatándose su toxicidad a dosis elevadas en monos y perros, en los que llegó a producir convulsiones y muerte (Kirsch, 1986). Dichos ensayos fueron realizados en Ann Arbor por la Universidad de Michigan por encargo del Edgewood Chemical Warfare Service (U.S. Army) y sus resultados no fueron publicados hasta años más tarde, concretamente en 1973 (Shulgin, 1986).

No debemos olvidar que en aquella época los grupos de militares americanos estaban sumamente interesados en hallar nuevas "armas" químicas que fuesen aplicables en el contraespionaje y los psicodélicos parecían constituir unos agentes muy adecuados. No obstante no existen datos que avalen que el MDMA fuera utilizado con tales fines (Shulgin y Shulgin, 1992).

Años más tarde, en 1965, Alexander Shulgin, que por aquel entonces trabajaba para la Dole Company, resintetiza la MDMA, y a partir de aquel momento comienza a ensayar con la sustancia y difundirla entre personas cercanas a él, siendo una de ellas, en 1967, el psicólogo Leo Zoff .

Zoff, que había desarrollado a lo largo de su ejercicio profesional el uso terapéutico de determinadas sustancias psicodélicas como la MDA, el LSD y la ibogaína, se interesa a partir de aquel momento por el posible potencial terapéutico de la MDMA, dedicándose durante los últimos años de su vida (murió en 1977), a introducirla, bajo el nombre de Adam, en determinados círculos terapéuticos sobre todo del área de San Francis$\mathrm{co}$, ya que las leyes de este estado permitían el uso terapéutico de productos no comercializados siempre que fueses preparados y administrados in situ por un médico o farma- 
céutico (aunque no se sabe con exactitud, se supone que al menos 15 profesionales utilizaron la MDMA con fines terapéuticos en el periodo comprendido entre 1970 y 1985) (Eisner, 1995).

No obstante, no es hasta 1976 cuando Alexander Shulgin y su colaborador David Nichols publican el primer informe sobre los efectos psicoactivos de la MDMA en el hombre (Shulgin y Nichols, 1978). Posiblemente, a este hermetismo contribuyó en gran medida el hecho de que los profesionales que la utilizaban con fines terapéuticos tenían miedo a que sucediese algo similar a lo que había ocurrido anteriormente con el LSD, que tras la difusión pública de sus efectos psicoactivos, pasó de ser un instrumento de ayuda para los psicoterapeutas a ser una droga ampliamente difundida entre los integrantes de la contracultura "hippy", y a partir de ese momento fue rápidamente ilegalizada (Eisner, 1995).

El tráfico de MDMA, aunque todavía a muy pequeña escala, había comenzado unos años antes, ya que en 1970 un grupo formado por químicos y pequeños empresarios comenzaron a fabricar MDMA en un laboratorio situado en Marin County (California) (Roig-Traver, 1994). Se dice que el primero en utilizar el nombre de éxtasis fue un productor clandestino de San Francisco, que en realidad quería llamarla empathy, dados los efectos de la sustancia, pero se dio cuenta de que el nombre de "éxtasis" funcionaba mejor desde el punto comercial (Bagozzi, 1996). Mientras que Adam había sido un instrumento terapéutico y experimental, el éxtasis iba a ser un recurso lúdico para participar en "fiestas", favorecer las relaciones sociales y pasarlo bien (Beck y Rosenbaum, 1994). Estas dos etiquetas no sólo reflejaban diferencias en los motivos para ingerir la sustancia, sino que implicaban diferencias en el contexto y la frecuencia de uso, las dosis, la combinación con otras drogas y las redes de distribución de la mercancía (Gamella y Alvarez, 1999).

En aquel momento la MDMA se distribuía acompañada de folletos en los que informaba acerca del modo adecuado de consumirla sin riesgos ("Ecstasy, everything looks wonder- ful when you're young and on drugs", "How to prepare for an ecstasy experience," "Flight intructions for a friend using XTC", "Ecstasy: 21st Century entheogen"), aconsejándose de igual modo, no asociar el consumo de MDMA al de otras sustancias, no usarla durante largos periodos de tiempo, etc (RoigTraver, 1989).

Este laboratorio fue poco a poco incrementando su producción, de modo que en 1985 producía unas 500.000 dosis al mes y estaba disponible, amén de en California, en otros 21 estados fundamentalmente de la costa este, además de Canadá, de igual modo, se inicia por aquel entonces la distribución a Europa (Kirsch, 1986).

Por otra parte, durante 1976 un grupo de químicos de Boston comenzó a producir MDMA en cantidades relativamente pequeñas y se apoderó de su mercado y tráfico hasta comienzos de los ochenta (Capdevila, 1995).

A comienzos de los ochenta el consumo de MDMA comenzó a experimentar una cada vez más creciente popularización, convirtiéndose a partir de 1983 en la droga sacramental del movimiento New Age. Este grupo producía la sustancia y la distribuía junto con una Guide for Users, que en siete páginas describía los aspectos farmacológicos del MDMA, sus modos de administración, sus contraindicaciones y hacía una serie de sugerencias generales. Los nombres con los que más popularmente se conocía a la sustancia eran en aquel momento: ecstasy o XTC, sin olvidar los ya mencionados al comienzo del capítulo (Escohotado, 1992).

No era infrecuente que se facilitase también, la fotocopia de un artículo de Timothy Leary, en el que la MDMA era considerada la "droga de la década". El mayor inconveniente de la sustancia, según palabras de Leary, residía en el "síndrome del matrimonio instantáneo", ya que la fuerza emocional de algunas experiencias con MDMA podía potenciar relaciones tan intensas que llevasen casi de forma inmediata al altar. No siendo por tanto descabellado, que por aquella época (años ochenta), comenzaran a aparecer en los cam- 
pus de algunas universidades americanas camisetas portando el slogan "Don't get married for 6 weeks after XTC" (Eisner, 1995).

Por aquel entonces la MDMA era distribuida mucho más ampliamente e incluso comenzó a venderse en bares (principalmente de Dallas y Austin -Texas-), donde se aceptaba el pago mediante tarjeta de crédito, a la vez que comenzaron a aparecer carteles anunciando la realización de "fiestas de éxtasis". La MDMA estaba comenzando a convertirse en la última moda en lo que a drogas se refiere y comenzaba a seducir a todo tipo de gente.

El período comprendido entre los años 1977 y 1985 puede considerarse como la edad de oro de "Adam". Durante esa época los usuarios no tienen nada que ver con los actuales y el número de personas que la utilizan es todavía escaso. Cabría citar, por un lado, el uso en círculos terapéuticos y por otro, estaría el uso experimental dentro de ambientes contraculturales de la época (new agers), que lo utilizan por sus propiedades expansoras de la mente. A finales del año 1984, siendo aún una droga completamente legal, comienza a introducirse en ambientes estudiantiles y en los ambientes frecuentados por los "young urban professional people" (yuppies), que si bien utilizan en más cuantía la cocaína, no desdeñan en absoluto las posibilidades de esta sustancia (Bagozzi, 1996).

Durante esa época, se distinguen, en Estados Unidos, dos tipos básicos de redes de distribución de la sustancia, que tenían visiones distintas de la misma y trabajaban para públicos diferentes. Por un lado, estaban los vendedores minoritarios que ofrecían la MDMA a conocidos y estaban guiados por un propósito social y no sólo económico. Por otro, fueron apareciendo "empresas" orientadas al provecho rápido mediante el comercio anónimo y organizado, aunque no tan criminalizadas como las que dominaban el mercado de cocaína o heroína. Los primeros, más elitistas, pretendían restringir su uso a círculos selectos de consumidores; los segundos, más proselitistas, querían ampliar su clientela y difundirse entre todos los públicos. De este modo, se fue desarrollando un sistema piramidal de organización que consiguió multiplicar el número de detallistas y el éxtasis se volvió mucho más accesible al público (Gamella y Alvarez, 1999).

Durante este período existen, en Estados Unidos, cuatro "universos sociales" dónde se popularizó mayormente esta droga: estudiantes universitarios, jóvenes (y no tan jóvenes) profesionales o yuppies, homosexuales y, new agers. Además, existe una rápida expansión del consumo de éxtasis en tres escenas populosas: entre los deadheads, seguidores de la banda de rock psicodélico Greateful Dead, entre los "festeros" noctámbulos en Dallas (Texas) entre 1983 y 1985 y, más recientemente, entre los ravers contemporáneos que, surgidos en Europa, han influido en el desarrollo de una escena norteamericana de "fiestas" multitudinarias basadas en la música techno (Gamella y Alvarez, 1999).

\section{CRIMINALIZACION DE LA MDMA}

El hecho de que entre los años 1977 y 1985 prácticamente no existiesen casos de toxicidad asociados al consumo de MDMA (entre 1977 y 1981 hay descritos 8 casos de personas que necesitaron tratamiento de urgencia tras haber consumido MDMA y ninguno entre 1981 y 1985), es quizás uno de los motivos por los que la legislación norteamericana fue permisiva con el consumo de esta sustancia. No obstante ante los datos referidos por la Organización Mundial de la Salud (OMS) en 1984, que ponen de manifiesto que la MDMA es la única feniletilamina incautada en varias ocasiones, la Drug Enforcement Agency (DEA) publica un aviso en el Federal Register del 27 / 07 / 84, en el que propone incluir la MDMA en la Lista I de la Comprehensive Substances Act, lo cual se traduciría en expresar que la MDMA estaba dotada de un alto potencial de abuso, carecía 
de uso médico conocido e incumplía las normas de seguridad exigidas por la Food and Drug Administration (FDA).

La respuesta pública a la decisión de la DEA no se dejó esperar, ya que el 10 de septiembre de 1984, un grupo compuesto por diferentes profesionales (médicos, psicoterapeutas y abogados) y que atendía al nombre de Earth Metabolic Design Laboratory, envió una carta por medio de su abogado (Richard Cotton) al administrador de la DEA (Francis Mullen), solicitando la celebración de una reunión para determinar si la MDMA sería incluida en alguna lista y en caso positivo, en que lista.

La primera reunión se celebró en las dependencias de la DEA en Washington ante el juez Francis Young, el 1 de febrero de 1985 y se decidió que se celebrarían tres reuniones más (en Washington, Kansas City y Los Angeles, respectivamente). El grupo defensor de la MDMA alababa la capacidad terapeútica de la sustancia, la consideraban una sustancia suficientemente segura para el uso médico [el estudio realizado por Downing en 1984 (Downing, 1986), practicamente no encontraba anormalidades remarcables en un grupo de 21 sujetos que habían consumido MDMA], y solicitaba la inclusión de la MDMA en una lista distinta de la I, lo cual facilitaría su eventual empleo terapéutico y por otra parte, permitiría la persecución del tráfico ilegal de dicha sustancia.

La polémica había alcanzado la calle y diversos medios de comunicación americanos se hicieron eco de ella. Así, el 15 de abril de 1985, el Newsweek publica en su sección Vida/Estilo, un artículo titulado "Viajar con el "Extasis" " y posteriormente otras revistas como Time, New York Magazine y Life, entre otras, publican reportajes sobre la MDMA a lo largo de 1985. Y, por si fuera poco, a comienzos de dicho año (1985), se detecta un súbito aumento de la producción de MDMA en un laboratorio de Texas que pasa a producir unas 8.000 dosis al día y cuenta con unas redes de distribución de la sustancia cada vez más amplias, tanto a nivel nacional como internacional.
Gracias a los poderes especiales concedidos a la DEA por el Congreso de los Estados Unidos, mediante la Comprehensive Crime Control Act, también conocido como el Emergency Schedulling Act, de 1984, tras las muertes y graves lesiones neurológicas producidas en algunos consumidores del opiáceo conocido como china white (fentanilo), ésta tiene capacidad para incluir por procedimiento urgente cualquier sustancia en la Lista I durante un período de un año, y, ésto es lo que hace, ya que el 31 de mayo de 1985, la DEA anuncia la inminente inclusión de la MDMA en la Lista I de la Comprehensive Substances Act, basándose sobre todo en un estudio realizado por Charles Schuster publicado en la revista Science, que manifestaba que la MDA era capaz de producir lesión cerebral en ratas.

A pesar de las críticas recibidas por el informe de Schuster y de las alegaciones realizadas entre otros por David Nichols, June Riedlinger y Alexander Shulgin, a favor de la MDMA, el 1 de julio de 1985, la DEA incluye a esta sustancia en la Lista I de la Comprehensive Substances Act, quedando prohibido su consumo con fines terapéuticos o recreativos y su tráfico ilegal.

Aproximadamente un año más tarde, el 22 de mayo de 1986, Francis Young, juez de la DEA especialista en derecho administrativo y persona que había actuado como presidente de las reuniones sobre su ilegalización, presenta un informe de 90 páginas, en el que recomienda que la MDMA sea incluida en la Lista III, lo cuál permitiría el empleo con fines médicos de la sustancia. No obstante, el 17 de junio de 1986, la DEA amplía durante otros 6 meses la inclusión del MDMA en la Lista I, inclusión que se torna permanente desde el 13 de noviembre de 1986.

Poco después, en septiembre de 1987, se acepta una apelación presentada por Lester Grinspoon y, el tribunal de apelación del primer distrito ordena a la DEA, el 18 de septiembre de 1987, que retire al MDMA de la Lista I, argumentando que la DEA no había seguido un criterio correcto, desde el punto de vista jurídico, en la decisión tomada sobre 
si la MDMA cumplía o no las normas de seguridad comúnmente aceptadas para su uso médico. De igual modo, recomienda su inclusión en la Lista III. Dicha sentencia se hace efectiva el 27 de enero de 1988, pero la DEA contraataca, corrigiendo el procedimiento administrativo y consigue que desde el 23 de marzo de 1988 la MDMA esté incluida bajo el código n 7405 de la Lista I de la Comprehensive Substances Act.

Desde el momento en que la DEA incluye a la MDMA en la Lista I de la Comprehensive Substances Act el 1 de julio de 1985, el sector disconforme con dicha inclusión se centra en tratar de conseguir que la OMS, permitiera la fabricación legal y la utilización terapeútica de la sustancia (no hay que olvidar que los paises miembros de la ONU son signatarios de la International Convention on Psychotropic Substances -ICPO- y se rigen por las recomendaciones del Comité de Expertos en Drogodependencias de la OMS). No obstante, en la XXIII Reunión del mencionado Comité de Expertos, celebrada en Ginebra del 22 al 26 de abril de 1986, el Comité estuvo de acuerdo en incluir la MDMA en la Lista I de Convenio sobre Psicotrópicos celebrado en Viena el 21 de febrero de 1971, convirtiéndose la MDMA en una droga ilegal en todo el mundo.

A partir de ese momento, los acontecimientos se desencadenan de un modo rápido y el 22 de abril del mismo año (1986), la MDMA es considerada como droga ilegal en Suiza; el 18 de julio en Alemania, y en Italia en el año 1988. En Gran Bretaña la prohibición era anterior y databa de 1977, al igual que ocurría con el resto de anfetaminas psiquedélicas, incluyendo MDA y MDEA (Bagozzi, 1996).

El Convenio sobre Psicotrópicos firmado en Viena entró en vigor en España en el año 1976 (BOE 10 de septiembre de 1976). En lo que a la MDMA respecta, España acató la decisión de la OMS al mes siguiente, de modo que una orden ministerial aparecida en el BOE del 30 de mayo de 1986, incluye a esta sustancia en el Anexo I del Real Decreto 2829 del 6 de octubre de 1977, en el que se regulan las sustancias psicotrópicas de acuerdo con la propuesta del Convenio de Viena, quedando así prohibido el uso, fabricación, importación, tránsito y comercio de la MDMA, así como de todos los preparados que la contengan.

No obstante, recientemente parece que se están realizando algunos cambios, así por ejemplo, las leyes suizas sobre narcóticos incluyeron en 1988 una exención especial que permite a médicos especialmente formados recetar MDMA y otros compuestos psicoactivos. De ese modo, en el período comprendido entre 1988 y 1990, se permitió a un pequeño grupo de psiquiatras, la administración de MDMA a sus pacientes sin restricciones. No obstante, en 1990 el gobierno suizo se ve obligado a tomar medidas tras un penoso incidente, el fallecimiento inesperado de que un paciente tras la administración de ibogaína. Desde 1990 a 1992 se detuvo el uso de MDMA en Suiza y, en 1992, una vez que se determinó que la MDMA no había tenido nada que ver con la mencionada muerte, se vuelve a permitir trabajar con esta sustancia, pero sólo en aquellos pacientes que hubieran sido tratados previamente con MDMA y no hubieran hechos progresos suficientes (Eisner, 1995).

De igual modo, las autoridades sanitarias suizas han permitido la realización de un estudio farmacocinético con la MDMA, en sujetos previamente tratados con esta sustancia. Este estudio lo llevó a cabo por Hemlin del Instituto Farmaceútico de la Universidad de Berna (Eisner, 1995).

Por otro parte, en Estados Unidos, la Sociedad Multidisciplinaria de Estudios Psicodélicos (MAPS), fundada por Rick Doblin, ha continuado con su lucha particular, y tras un largo proceso han conseguido que la FDA aprobase en 1992, un estudio sobre la seguridad del empleo de MDMA en humanos (fase 1). Dicho estudio ha sido llevado a cabo por el Dr. Charles Grob en el Harbor-UCLA Medical Center, perteneciente a la Universidad de California. Dicho estudio ya ha sido finalido y publicados los resultados del él extraídos durante 1996. 
Actualmente, se han realizado estudios sobre seguridad y efectos neurofisiológicos inducidos por la MDMA en personas en distintos países, entre ellos España (concretamente, en nuestro país, se han realizado este tipo de estudios en el Instituto Municipal de Investigación Médica -IMIM- de Barcelona).

\section{EXPANSION DEL FENOMENO DE USO-ABUSO DE MDMA}

¿Cómo pasa el éxtasis de ser una droga de uso bastante restringido a ser la "droga para bailar"? Desde unos contextos de uso bastante específicos, la MDMA se va poco a poco filtrando en los clubs más exclusivos de Chicago, New York, San Francisco y Detroit, de la mano de los yuppies metropolitanos sumidos en el glamour del mundo del arte, del cine y de la música. La MDMA encuentra el mejor terreno en las warehouse parties, fiestas muy de moda en almacenes abandonados y en clubs predominantemente gays, donde se comienza a hacer experimentación musical. En Chicago, es el club Warehouse, paraiso de homosexuales y afroamericanos, en Nueva York sería el Paradise Garage. En estos locales, el los albores de los años 80, dos disc jockeys americanos, Frankie Knuckles "el Padrino" en Chicago y Lerry Levan en New York, comienzan a crear un nuevo estilo musical con la intención de cubrir el vacío que se estaba creando en mundo de la música (Bagozzi, 1996).

Estos serían los primeros pasos de lo que posteriormente se conoció como house music. House porque, según la versión del disc jockey inglés Richard West conocido como Mr. C, se escuchaba en el Warehouse de Chicago. Sin embargo, versiones menos refinadas, refieren que el término house procedería del hecho de que este tipo de música puede ser fácilmente producida en cualquier casa, siempre que se disponga, eso sí, de la tecnología necesaria. La leyenda dice, asimismo, que el Paradise Garage de New York habría dado nombre a un subgenero de la musica house, la garage (Bagozzi, 1996).

El house rompe con todos los moldes musicales existentes hasta la fecha, lo mezcla todo, especialmente $r \& b$, música latina, hip hop, funky y la música electónica alemana de comienzos de la década de los años 80. La única concesión que tecnología hace a esta mezcla completamente electrónica es la presencia de la voz del cantante, generalmente femenina (Bagozzi, 1996).

Descendiente directa de la house es la techno, variante totalmente computarizada de unos 140-160 beats por minuto. A esta nueva familia se irían uniendo la extrema hard core y gabber (200 beats por minutos), la tribal, la ambient y la hipnótica trance, así como la igualmente batiente e imprevisible progressive (Bagozzi, 1996).

La palabra rave es un término surgido para hacer referencia, desde hace algunos años, a un tipo de fiestas multitudinarias que se celebran en espacios cerrados, que se prolongaban durante toda la noche $y$, en las que predominaba un tipo de música fuerte y repetitiva que usaba muestras de música previamente registrada, que eran mezcladas a modo de collage y que fue apodada como acid house y, que generalmente se acompañaba de proyecciones de vídeo generadas por ordenador y proyecciones de láser.

El origen del término acid house no está del todo claro, ya que si bien algunos autores consideran que proviene del argot existente en las calles de Chicago dónde significaba robar (pretendiendo hacer referencia al proceso de creación de esa clase de música), son muchos más numerosos los que piensan que hace referencia al nombre coloquial del LSD, droga consumida preferentemente en las primeras fiestas acid house (Robles, 1993).

Existen fuentes (Lles, 1994), que apuntan a que fue en Chicago donde comenzó, en torno a 1988, el movimiento acid house. Coincidiría con el hecho de que algunos disc jockeys comenzaron a reunir a grupos de jóvenes en un lugar denominado the house, donde permanecían bailando durante toda la 
noche un tipo de música denominada early o garage house, que consistía en fragmentos de discos que eran copiados y mezclados electrónicamente por medio de un sampler o muestreador.

Desde América, house, techno y éxtasis atraviesan el océano y penetran en el Viejo Continente, primero Ibiza y Valencia, posteriormente, Londres y Manchester. Estamos a mediados de los años 80 y en Ibiza comienzan a hacer furor en dos de las más famosas discotecas a nivel europeo de la época, Amnesia y Pachá. Sin embargo, en Londres, el comienzo sería en locales gay como el Shoom, el Pyramid, el Jungle, donde se bailaba house toda la noche y se comenzaba a utilizar las primeras dosis de MDMA (Bagozzi, 1996).

La mayoría admite que fue España, concretamente Ibiza, el lugar desde donde se exportó al resto del mundo este tipo de fiestas y el movimiento acid house. En el comienzo de los años ochenta, Ibiza constituía el lugar de encuentro habitual tanto de vendedores de drogas como de seguidores del movimiento denominado New Age (movimiento espiritualista para el que la MDMA parecía constituir la droga sacramental), por lo que alrededor de 1985, la MDMA circulaba con cierta difusión por la isla, fechándose el inicio de los raves en el año 1987.

Es en esa época cuando la juventud inglesa que veranea en Ibiza, comienza a importar ese tipo de fiestas a su país, dónde se convierten junto con el consumo de éxtasis en uno de los fenómenos de mayores repercusiones sociales de los últimos años. Concretamente, a finales del 87 y comienzos del 88 se aviva la tendencia en Inglaterra, cuando un grupo de cuatro disc jockeys ingleses -los "cuatro jinetes del apocalipsis"-, Paul Oakenfold, Johnny Walker, Nicky Holloway y Danny Rampling, regresan de Ibiza tras haber visitado Amnesia y Pachá y deciden recrear en su país lo vivido en estos lugares. En sus clubs sonaban exclusivamente discos que pertenecían a un tipo de música aún inédito, pero que posteriormente se haría famoso con el nombre de acid house. La prensa se hace por primera vez eco del fenómeno a finales de junio de 1989, cuando a la mañana siguiente de la inaguración de la discoteca Trip, en Londres, cientos de jóvenes continuan bailando en la calle, gritando con todas sus fuerzas la palabra acid. Su aspecto era totalmente calcado de las fiestas baleares: bandana o foulard en la cabeza a modo pirata, gafas de sol en plena noche, vaqueros gastados, zapatillas deportivas Converse y camisetas con el símbolo de Smiley, el muñeco amarillo de la gran sonrisa (Bagozzi, 1996).

Ante la proliferación cada vez mayor de las macrofiestas, en 1990, el Parlamento Británico decretó una ley (Entertainments Increased Penalties Act), que marca sanciones contra los raves cada vez más duras. Este aumento de las sanciones trajo como consecuencia que estos grupos fueran desplazándose de las ubicaciones tradicionales a lugares menos previsibles, generalmente grandes almacenes vacíos o campos abiertos que solían localizarse en las inmediaciones de la red de autopistas que circunvalan Londres (Orbital). Se solía mantener en secreto el lugar de la celebración de la fiesta, con el fin de que a la policía le resultase más difícil dar con su localización, no obstante, al ser éstas multitudinarias, no era fácil que pasaran desapercibidas.

De aquella época, se ha descrito que durante 1992, en las cercanías de Manchester, promotores sin escrúpulos llegaron incluso a cortar el suministro de agua de sus lavabos, a encender la calefacción y a apagar el aire acondicionado, con el fin de vender más bebidas (no olvidemos la deshidratación que produce el MDMA, a lo que se sumaba el calor reinante en los locales y el baile continuo) a grupos de jóvenes mermados de capacidad adquisitiva. Concretamente, uno de los locales en los que se practicaba tal costumbre alcanzó una triste notoriedad cuando salió a la luz pública que se habían visto en él a chicas bebiendo de la taza del water.

Como la presión ejercida por las autoridades inglesas era cada vez más agobiante un grupo de promotores y disc jockeys, no dudó en desplazarse a California y, el resultado fué que a principios de 1991, un grupo denomina- 
do Toontown, celebró en San Francisco una de las primeras macrofiestas. Poco a poco comenzó a no ser infrecuente que en ciudades como Los Angeles y San Francisco se celebrasen una docena de fiestas en el mismo fin de semana.

Poco a poco se fue gestando un gran cambio y a partir de las grandes fiestas clandestinas acid house de finales de los ochenta comienza a desarrollarse la cultura del ocio; así, a partir de 1988 se produce un cambio de estatus pasando de ser una cultura underground y elitista, a ser una cultura mucho más popular asociada al ocio y por todos conocida como movimiento techno. Dicho movimiento es quizá el más popular en nuestros días, como prueban los multitudinarios techno-carnavales alemanes, la ruta del bakalao española, la popularidad alcanzada por algunos locales como Omen en Frankfurt, Rex en Paris y Hacienda en Manchester, así como el fenómeno británico de los travellers (grupos de nómadas que viven en rulottes, desplazándose de un sitio para otro y reuniéndose en lugares poco convencionales para celebrar fiestas en el campo) (Capdevila, 1995).

La "tribu" de asistentes a las discotecas varían en función del tipo de música predominante en ella, y de igual modo varía el tipo de droga predominantemente usada. Así, en los locales dónde predomina la música techno hard core, gabber o progressive (las variaciones más "duras" de música techno con alrededor de 200 beats por minuto), MDMA, anfetaminas y cocaína son consumidas preferentemente. En aquellos en los que el tipo de música dominante es más "suave", techno trance o ambient, suele consumirse MDMA y LSD y por último en los locales donde predomina la música jumgle, la droga más utilizada es el hashish. No obstante, hay que tener presente que en cualquiera de los diferentes locales podría localizarse cualquier tipo de droga.

\section{EL FENOMENO EXTASIS EN NUESTRO PAIS}

A pesar de que ya existían publicaciones previas, en nuestro país la MDMA comienza a crear gran polémica, a partir del reportaje titulado "Las espinas del bakalao", publicado en 1993 en la revista Tráfico, perteneciente a la Dirección General de Tráfico. En él se hacía referencia a un itinerario que unía Madrid y Valencia y por el que circulaban miles de jóvenes todos los fines de semana. Estos jóvenes eran capaces de conducir gran cantidad de kilómetros, moviéndose de discoteca en discoteca, y era usual entre ellos el consumo de MDMA y otros análogos. Poco después, en noviembre de 1993, el Ministerio del Interior anuncia medidas para "prevenir y reprimir" las rutas del bakalao (Capdevila, 1995).

Aunque los raves y la ruta del bakalao son considerados por la mayoría de los jóvenes simplemente una forma diversión, existe al menos un pequeño grupo de fieles usuarios que dotan al fenómeno de cierto ambiente de culto, lo cual no es de todo extraño si se tiene en cuenta la situación: el disc jockey, gran maestro de ceremonias, como un sumo sacerdote o un chamán electrónico dirige sabiamente lo que acontece en la "catedral" (discoteca), controla la música, se encarga de la proyección del láser que está sincronizado con el sonido y, mientras tanto la pantalla de video proyecta imágenes en vivo de la multitud. Al mismo tiempo la multitud de jóvenes bailan al trepidante ritmo de la música y el disc jockey dirige con frases adecuadas la ceremonia (en este sentido casi todos podemos recordar el slogan: "Exta-si, Exta-no, Esta me la como yo...," hecho famoso por un disc jockey valenciano).

A las diversas rutas del bakalao existentes en nuestra geografía pasa a añadirse un nuevo ingrediente, los afters hours, locales que abrirían a primeras horas de la madrugada y donde disc jockeys y resto de allegados a esta moda podrían seguir oyendo y bailando música "máquina" hasta el mediodía del día siguiente. En este sentido, cabe comentar, que para tratar de eludir la persecución poli- 
cial, algunos de estos locales after hours, cuentan con permiso de croisantería, lo que les permitiría abrir a primeras horas de la mañana (Capdevila, 1995).

En la expansión del fenómeno éxtasis, en nuestro país, pueden distinguirse cinco períodos bien diferenciados (Gamella y Alvarez, 1999): fase previa, fase inicial, fase de popularización, fase de vulgarización y consumo masivo y fase de rutina y agotamiento del ciclo o fase de estabilización.

\section{Fase previa (1978-1986)}

Desde finales de los 70 tenemos noticias de la presencia de estas drogas en ciertos entornos turísticos de nuestro país, sobre todo en Baleares. Estamos ante un consumo muy puntual, experimental, generalmente, realizado de la mano de norteamericanos o europeos que habían conocido la sustancia en Estados Unidos. Los primeros consumos grupales ocurren en Ibiza en 1983, siendo consumos todavía muy restringidos. Por otra parte, entre 1984 y 1986 se constata la presencia del éxtasis en otras zonas como Madrid, Barcelona, Marbella y, sobre todo, Valencia (Gamella y Alvarez, 1999). A mediados de los 80 ya existía en Valencia una red de locales y discotecas (Cacao, Spook Factory y, posteriormente, AC/TV, Masía, Puzzle, N.O.D., Zona, etc) con horarios encadenados que ofrecían sesiones ininterrumpidas, sin límites horarios, durante los fines de semana (Usó, 1996).

\section{Fase inicial (1987-1989)}

En esta fase el consumo seguía centrado en ciertos entornos cosmopolitas, elitistas y contraculturales, que adquirían la sustancia en otros países. Ibiza sigue desempeñando un papel fundamental en la expansión de la sustancia, comenzándose un tráfico incipiente con otras islas Baleares, Madrid, Cataluña y Valencia. En estos años se produjeron también las primeras incautaciones policiales de MDMA (en 1986 se aprehenden en Ibiza las dos primeras cápsulas de éxtasis y aparece el primer reportaje en la prensa española en relación con la sustancia ("Pólvora de amor y éxtasis", 30 de agosto de 1987, El País). Este momento coincide con la llegada a España de la música acid house que fue adaptándose al mercado español y derivando en otros estilos entre los que destaca el bakalao (Gamella y Alvarez, 1999).

En estos momentos el éxtasis era todavía poco asequible en España y en la mayoría de casos era importado por jóvenes españoles y extranjeros que trabajaban por su cuenta y a pequeña escala.

\section{Fase de popularización (1990-1991)}

En esta etapa el consumo de éxtasis se extiende a amplios sectores de la población juvenil, popularizándose más allá de los ambientes bohemios, contraculturales o incluso festeros y bakaladeros. Al principio aparece, sobre todo, en zonas urbanas y turísticas (Baleares, Levante, Cataluña, Madrid y algubos puntos del País Vasco) y va penetrando hacia el sur y el interior de la península. En esta época se producen también las primeras emergencias médicas asociadas al uso de estas pastillas (El País, 1 de agosto de 1991) (Gamella y Alvarez, 1999).

\section{Fase de vulgarización y consumo masivo (1992-1996)}

En esta fase se alcanza el mayor crecimiento en el consumo de esta droga, aumentando enormemente su disponibilidad lo cual se refleja en la caída de su precio. El éxtasis se desideologiza y se convierte en un objeto de consumo casi convencional. Su uso se extiende a toda la geografía española, incluyendo entornos provincianos y zonas rurales, a la vez que se amplían las redes de su distribución. Los más jóvenes comienzan a usar estas sustancias (en su argot, pastillas, pastis, pirulas, etc) a edades cada vez más tempranas. En este período se produce la eclosión de las "rutas del bakalao" y se crea gran polémica en los medios de comunicación. En estos años se repiten los casos de intoxica- 
ción y se comunican los primeros casos de muertes en los que aparece implicada la MDMA (sola o en sinergia con otras drogas) (Gamella y Alvarez, 1999).

\section{Fase de rutina y agotamiento del ciclo o fase de estabilización (1997-)}

A pesar de seguir existiendo, sobre todo entre los adolescentes, deseo de probar la sustancia, el ritmo de personas que se han incorporado a su consumo parece haberse estabilizado, tal como demuestran las diversas encuestas poblacionales realizadas por el PNSD tanto en población general como en estudiantes de secundaria, así, como las realizadas en diversos lugares concretos de nuestra geografía. En este período se detecta una caída en la calidad del producto disponible en la calle y la creciente participación en su venta de grupos o clanes con experiencia en el tráfico de drogas ilegales (Gamella y Alvarez, 1999).

\section{NUEVAS TENDENCIAS ASOCIADAS AL FENOMENO EXTASIS}

Al lado de los tradicionales raves, se asistió al nacimiento de otro tipo de fiestas. Surgidas en la discoteca Velvet Underground de Londres en diciembre de 1994 y sin relación directa con la MDMA (aunque no se excluye su consumo), este nuevo estilo de fiestas fueron importadas a nuestro país por Javier Bellot, que los primeros viernes de cada mes comenzó a organizar en la discoteca Xenón de Madrid y bajo el nombre de Ritual, unas fiestas "angelicales", con un decorado paradisíaco (tules blancos, proyecciones manieristas y tenebristas, fragancias exóticas, alas de ángeles flotando sobre la pista de baile) y dónde se escuchan ritmos house, garage, trance, y cuyo fin era "subir las vibraciones del público hasta provocar su estado de felicidad" (Capdevila, 1995).

Por otro lado, no hay que olvidar el uso cada vez más frecuente de MDMA ligado a determinados acontecimientos sociales de tipo multitudinario no asociados al baile, tal como serían los partidos de futbol, los círculos de movilización política o incluso paramilitar de Irlanda del Norte o el fenómeno de los "techno travellers" (nómadas de la música techno) (Saunders, 1995). Así, Nicolas Saunders (1993), en su libro "E", comenta como el investigador inglés Mark Gilman fue testigo del paso de consumo de alcohol al de éxtasis entre los jóvenes supporters del Manchester City y del Manchester United, durante el la temporada 1991/92.

Desde 1989, año en que el Manchester City había vuelto a militar en la primera división del futbol inglés, los encuentros de máxima rivalidad entre ambos equipos siempre se habían saldado con importantes disturbios. Sin embargo, algo iba a ocurrir en el derby de 1991, que cambiaría radicalmente la situación. Según palabras de Gilman, la influencia de la MDMA fue decisiva a la hora de impedir las escaramuzas habituales entre ambos grupos de supporters, y los aficionados de ambos equipos compartieron la misma escena rave durante la noche anterior al partido sin que existiese ningún problema entre ambos bandos (Saunders, 1993).

Esta tendencia también se está observando en otros países como Italia, tal y como ponen de manifiesto los datos recientemente aportados por Schifano (1996), que en un trabajo llevado a cabo utilizando una muestra de 343 frecuentadores de discotecas en la región de Veneto, encuentra que el $6 \%$ de los encuestados refieren consumir éxtasis en los estadios de fútbol.

No obstante, tampoco conviene que se mitifiquen estos aspectos, ya que no hay que olvidar que existen personas en las que la ingestión de MDMA no produce actitudes pacíficas, y que además, la ingestión conjunta del éxtasis con otras anfetaminas, speed o cocaína puede incitar a comportamientos más violentos que apacibles (Capdevila, 1995). 


\section{POSICIONAMIENTO ACTUAL ANTE EL FENOMENO EXTASIS}

El la conferencia New Trends in Synthetic Drugs, celebrada los días 18-19 de Noviembre de 1996 en Lisboa bajo los auspicios de la EMCDDA, se propone que sería necesaria la clarificación de los siguientes puntos en relación al creciente consumo de MDMA:

- ¿Cuales son realmente los efectos neurobiológicos a largo plazo?

- ¿Qué papel desempeña la susceptibilidad personal en la aparición de reaciones adversas? ¿Existen reacciones idiosincráticas?

- ¿Qué papel juegan los factores contextuales relacionados con el uso en los riesgos?

La clarificación de estos puntos, no está exenta de problemas. Así, por un lado estaría el hecho de que la mayoría de estudios epidemiológicos sólo reportan prevalencia vida, lo que da lugar a elevadas proporciones, por lo que sería de suma importancia que los estudios fuesen capaces de proporcionar información sobre el uso reciente; y, por otro, la necesidad cada vez más acuciante de realizar estudios que aporten luz respecto a los efectos toxicológicos en humanos a partir de investigaciones neuroendocrinas, funcionales y neuroquímicas.

Además, ante el creciente aumento experimentado en el consumo de MDMA, a pesar de las campañas abstencionistas, se van desarrollando con cada vez más ahínco campañas dirigidas a un aminoramiento de daños.

Así, aunque en un primer momento, las autoridades reaccionaron ante los eventos de danza con restricción, tomando medidas tales como cerrar los clubs, la tendencia actual es hacia la cooperación entre los servicios sociales y de salud, la policía y los organizadores y usuarios de los raves, con el fin de disminuir al máximo las consecuencias adversas debidas al consumo de MDMA y otras drogas. Esta colaboración puede ser tanto a nivel nacional, pero más a menudo lo es a nivel municipal.
Estos estamentos preocupados por el incremento de los problemas asociados con las drogas sintéticas han comenzado a editar material con información destinada a disminuir los posibles daños producidos por estas sustancias, así como con los riesgos que su consumo lleva asociado. Incluso dentro de lugares relacionados con su posible consumo, los organizadores de ese tipo de eventos informan acerca de los efectos nocivos, como golpe de calor, distrés psíquico, accidentes y problemas relacionados con el excesivo consumo y mezcla de drogas.

En múltiples países europeos, principalmente en el Reino Unido, Holanda, Alemania y Dinamarca, se ha distribuido ampliamente información de tipo preventivo, bien por medio de panfletos (leaflets) o en revistas de musica y life style o incluso en Internet. Es necesario señalar que, en nuestro país, existen diferentes entidades autonómicas que ya han dado los primeros pasos en lo referente a la publicación de folletos informativos relacionados con el consumo de esta sustancia. Incluso, en algunos países, el techno styles y la música se han utilizado para promover dancing libres de drogas alternativos (para revisión de estrategias de tipo preventivo dirigidas a los jóvenes europeos, ver Knutagard, 1995).

Fruto de la colaboración entre servicios sociales y de salud, organizadores de raves y usuarios es el hecho de que ayuda y primeros auxilios son facilitados en caso de accidentes - problemas psicológicos en los dance events en algunas localidades, sobre todo en los países anteriormente señalados.

Así, en Gran Bretaña y, tras incidentes tan escabrosos como el acontecido en 1992 en Manchester, ya comentado con anterioridad, se inició bajo el nombre de Safe Dancing Campaign una campaña en la que las autoridades obligaban a los clubs a cuidar unos mínimos para evitar riesgos asociados al consumo de MDMA: temperatura y calidad del aire ambiental, zonas más tranquilas donde poder descansar, suministro adecuado de agua, e incluso la realización de primeros auxilios básicos por parte de los empleados. 
En Holanda existe una organización denominada Safe House, que cuenta con una subvención gubernamental, dónde cualquiera (policía o consumidores) puede acudir a analizar sus pastillas. Safe House, también monta su tenderete en las macrofiestas, dónde por unas 200 pesetas (dos guilder) y en aproximadamente 2 minutos puede determinarse la pureza de las mismas. Cuando una remesa no está dotada de los "niveles de seguridad" adecuados, la organización distribuye panfletos en los que se describe el tipo de pastillas y se advierte del peligro que conllevan.

\section{EUROPEAN MONITORING CENTRE FOR DRUGS AND DRUG ADDICTION}

Por último, creemos obligado antes de la finalización de este capítulo hacer una breve referencia a qué es y cuáles son los objetivos del European Monitoring Centre for Drugs and Drug Addiction (EMCDDA). Dicha entidad con sede en Lisboa, fue fundado en esa misma ciudad, Lisboa, en el año 1994 y su misión consiste en recopilar y divulgar información respecto a los siguientes aspectos fundamentales concernientes a las drogas en los 15 estados miembros de la Unión Europea (EMCDDA, 1996):

- La demanda de drogas y las medidas encaminadas a reducir dicha demanda

- Las estrategias y políticas respecto a drogas, tanto a nivel nacional como a nivel comunitario

- La cooperación internacional y la geopolítica del suministro de drogas

- El control del tráfico de narcóticos, sustancias psicotrópicas y sustancias químicas precursoras

- Las repercusiones del fenómeno de la droga en los países productores, consumidores y de tránsito

Este organismo trabaja regularmente en contacto con otras organizaciones de caracter internacional, y las seis con las que la cooperación es más estrecha son las siguientes:
- El United Nations International Drug Control Programme (UNDCP), fundado en Austria, y que se encarga de la coordinación de todas las actividades relacionadas con las drogas realizadas por las Naciones Unidas. Los signatarios de dicha organización están obligados a proporcionar la información referente al uso-abuso de drogas (no se cubren datos referentes a drogas legales como el alcohol y el tabaco), que son recogidos a través de un cuestionario anual y que constituyen la base de los informes realizados por dicha organización, que incluye un informe global anual sobre el uso-abuso de drogas.

- La Regional Office for Europe of the World Health Organisation (WHO-Europe), fundada en Dinamarca, recoge y analiza los datos relacionados con aspectos de salud de los estados miembros, contando con un sistema específico de recogida de datos tanto de drogas legales como ilegales.

- El Cooperation Group to Combat Drug Abuse and Illicit Trafficking in Drugs, más conocido como el Pompidou Group, fundado en Francia, es una estructuctura intergubernamental dentro del Consejo de Europa. Entre sus miembros se encuentran los 15 países que pertenecen a la Unión Europea amén de otros países de Europa Central y del Este. Quizás una de las funciones más importantes de dicho grupo radica en realizar las mejoras metodológicas necesarias para facilitar la comparación de datos a nivel internacional.

- La Europol's Drugs Unit (EDU), que fundada en Holanda, comienza a desarrollar su trabajo en 1994 y forma parte de la European Police Office (Europol). Dicho organismo trabaja, sobre todo, el tema relacionado con el estado actual de producción y tráfico de drogas en la Unión Europea.

- La International Criminal Police Organisation (ICPO o Interpol), fundada en Francia, y cuyo objetivo básico consiste en el fomento de la cooperación entre las policías de los distintos países con el fin de combatir el crimen internacional. Dicha organización, crea en los años 70 una Sub- 
división de Drogas que centraliza y analiza los datos procedentes de sus estados miembros en lo referente al tráfico de drogas. Interpol lleva años cooperando con organizaciones internacionales como la UNDCP, la WHO, la WCO, el Consejo de Europa y la Comisión Europea, y desde 1995 con la EMCDDA.

- La World Customs Organisation (WCO), fundada en Bélgica, cuya principal objetivo es la publicación de un informe anual acerca de las aprehensiones de drogas realizazadas a nivel internacional. Los sofisticados sistemas de bases de datos con los que cuenta, a las que se puede aceder electrónicamente son actualizadas mensualmente, y son de gran utilidad no sólo para un mejor conocimiento de las diferentes rutas de tráfico de drogas, sino para un mejor conocimiento de las tendencias del tráfico ilegal de sustancias.

\section{CONCLUSIONES}

En pocas líneas vamos a tratar manifestar cuál es nuestro posicionamiento en torno al estado actual del fenómeno del éxtasis y de igual modo tratar de explicar cual es nuestra intención al promocionar esta monografía.

En primer lugar, hay que decir que existen en efecto una serie de factores que dificultan el estudio de los trastornos asociados al consumo de drogas de síntesis -ver tabla 1(Bobes, 1995, 1998).
En segundo lugar, hay que decir que nuestro propósito fundamental al abordar el estudio de las sustancias entactógenas es el de contribuir a aclarar el estado actual de este fenómeno subcultural que tanto caldeamiento social ha provocado en la última década. Hemos querido contribuir desde la psiquiatría y la psicología clínica a una mejor comprensión y conocimiento del consumo de estas sustancias, que parece obedecer a un estado de "anemia social" de algunos grupos de jóvenes.

Por otra parte, desearíamos contribuir a mejorar el enfoque médico-legal, aportando una visión más ajustada de la afectación conductual de estas sustancias, su verdadera capacidad de generar adicción, su impacto sobre la salud psicofísica y pública y su adecuada clasificación toxicológica (Bobes et al, 1994).

Comentar por último, que estamos completamente de acuerdo con las conclusiones obtenidas en la ya mencionada Conferencia de Expertos en New Trends in Synthetic Drugs (1996), acerca de que resulta necesaria una mayor y mejor información que haga referencia a la creciente diversidad de patrones de uso-abuso, a la intensidad y duración del uso de MDMA, a la combinación de drogas, drug careers, y referentes a las consecuencias que los usuarios pueden sufrir. Asímismo es necesaria más información sobre la prevención, disminución de riesgos y asistencia a usuarios, debiendo examinar, de igual modo, los métodos e indicadores más apropiados para identificar y monitorizar las nuevas tendencias y desarrollos (Bobes et al, 1998).

\section{Tabla 1: Factores asociados al consumo de drogas de síntesis}

1. Baja prevalencia de psicopatología, en relación con el alto índice de uso-abuso

2. Alta frecuencia de antecedentes personales y familiares de psicopatología

3. Alta frecuencia de psicopatología por uso-abuso de otras drogas

4. Gran diversidad en intensidad/tiempo de consumo de los casos observados

5. Elevada frecuencia de consumo concomitante o alternante de otras drogas (cannabis, cocaína, LSD, etc.), observándose una prevalencia prácticamente nula de consumidores puros

6. Relación temporal no bien establecida entre consumo y tiempo de aparición de la psicopatología

7. Ausencia de diferencias fenomenológicas entre los trastornos psicóticos esquizofrénicos y los trastornos de tipo psicótico aparecidos en relación con el uso-abuso de MDMA

Tomado de Bobes et al (1998) 


\section{REFERENCIAS}

1. Bagozzi F. Generazione in ecstasy. Torino: Edizioni Grupo Abele, 1996.

2. Beck J, Rosenbaum M. Pursuit of ecstasy: the MDMA experience. New York: State University of New York Press, 1994.

3. Bobes J, Sáiz PA, González MP, Bousoño M, Herrero S. Aspectos médico-legales del éxtasis (MDMA). Adicciones 1994; 6: 423-435.

4. Bobes J. Extasis. Aspectos farmacológicos, psiquiátricos y médico-legales. Barcelona: Neurociencias, 1995.

5. Bobes J, Lorenzo P, Sáiz PA. Extasis (MDMA): un abordaje comprehensivo. Barcelona: Masson, 1998.

6. Capdevila M. MDMA o el éxtasis químico. Barcelona: Los Libros de la Liebre de Marzo, SL, 1995.

7. Downing J. The psychological and physiological effects of MDMA on normal volunteers. J Psychoactive Drugs 1986; 18: 335-339.

8. Eisner B. Extasis. Historia del MDMA. Barcelona: Ediciones Obelisco, 1995.

9. EMCDDA. Annual report on the state of the drugs problem in the European Union. Lisboa: European Communities, 1996.

10. EMCDDA. New trends in synthetic drugs. Lisboa, Noviembre, 1996.

11. Escohotado A. Historia de las drogas 3. Madrid: Alianza Editorial, 1992.

12. Gamella JF, Alvarez A. Las rutas del éxtasis. Barcelona: Editorial Ariel, SA, 1999.

13. Grob Ch, Poland R, Chang L, Ernst T. Psychobiologic effects of 3,4-methylenedioxymethamphetamine in humans: methodological considerations and preliminary observations. Behavioural Brain Research 1996; 73: 103-107.

14. Holsten DW, Schieser DW. Controls over the manufacture of MDMA. J Psychoactive Drugs 1986; 18: 371-372.
15. Kirsch MM. Designer drugs. Minneapolis: Compcare Publications, 1986.

16. Knutagard $\mathrm{H}$. New drugs in Europe. A working document to be presented at the Pompidou Group seminar. June, 1995.

17. Lles LI. Cibersonidos: del futurismo al trip-hop. Rock de Lux 1994, Noviembre.

18. Merck E. Verfahren zur darstellung von alkyloxyaryl-dialyloxyaryl-und alkylenedioxyarylaminopropanen BZW. Deven am stickstoff monoalkylierten derivaten. Patente alemana 274.350, 1914.

19. Robles R. El día que murió el Acid House. Ajoblanco 1993, Febrero.

20. Roig Traver A. "El Extasis". Una revisión del MDMA, MDA y demás feniletilaminas psicoactivas. Actas de las XVII Jornadas Nacionales de Socidrogalcohol. Valencia, Diciembre, 1989.

21. Roig Traver A. Sobre el uso recreativo de la metilendioximetanfetamina: Aspectos históricos y efectos adversos. Addiciones 1994; 6 : 437-452.

22. Saunders N. E for ecstasy. London: Biddles of Guilford, 1993.

23. Saunders N: Ecstasy and the dance culture. London: Neal's Yard Studio, 1995.

24. Schifano F. Psychopathological consequences of MDMA abuse. International Conference on Ecstasy and Psychedelics: The State of the Art. Bolonia, 18-19 de Noviembre, 1996.

25. Shulgin AT. The background and chemistry of MDMA. J Psychoactive Drugs 1986; 18: 291 303.

26. Shulgin AT, Nichols DE. Characterization of three new psychotomimetics. En: The Psychopharmacology of Hallucinogens. Stillman R, Willete R (eds). New York: Pergamon Press, 1995.

27. Shulgin A, Shulgin A. Phikal. A chemical love story. Berkeley: Transform Press, 1992.

28. Usó JC. Drogas y cultura de masas (España 1855-1995). Madrid: Taurus, 1996. 
